\title{
BMJ Open Development and validation protocol for an instrument to measure household water insecurity across cultures and ecologies: the Household Water InSecurity Experiences (HWISE) Scale
}

To cite: Young SL,

Collins SM, Boateng GO, et al. Development and validation protocol for an instrument to measure household water insecurity across cultures and ecologies: the Household Water InSecurity Experiences (HWISE) Scale. BMJ Open 2019;9:e023558. doi:10.1136/ bmjopen-2018-023558

- Prepublication history and additional material for this paper are available online. To view these files, please visit the journal online (http://dx.doi. org/10.1136/bmjopen-2018023558).

Received 11 April 2018 Revised 19 0ctober 2018 Accepted 9 November 2018

Check for updates

(c) Author(s) (or their employer(s)) 2019. Re-use permitted under CC BY. Published by BMJ.

For numbered affiliations see end of article.

Correspondence to Dr Sera L Young; sera.young@northwestern.edu

\section{ABSTRACT}

Introduction A wide range of water-related problems contribute to the global burden of disease. Despite the many plausible consequences for health and well-being, there is no validated tool to measure individual- or household-level water insecurity equivalently across varying cultural and ecological settings. Accordingly, we are developing the Household Water Insecurity Experiences (HWISE) Scale to measure household-level water insecurity in multiple contexts.

Methods and analysis After domain specification and item development, items were assessed for both content and face validity. Retained items are being asked in surveys in 28 sites globally in which waterrelated problems have been reported (eg, shortages, excess water and issues with quality), with a target of at least 250 participants from each site. Scale development will draw on analytic methods from both classical test and item response theories and include item reduction and factor structure identification. Scale evaluation will entail assessments of reliability, and predictive, convergent, and discriminant validity, as well as the assessment of differentiation between known groups.

Ethics and dissemination Study activities received necessary ethical approvals from institutional review bodies relevant to each site. We anticipate that the final HWISE Scale will be completed by late 2018 and made available through open-access publication. Associated findings will be disseminated to public health professionals, scientists, practitioners and policymakers through peer-reviewed journals, scientific presentations and meetings with various stakeholders. Measures to quantify household food insecurity have transformed policy, research and humanitarian aid efforts globally, and we expect that an analogous measure for household water insecurity will be similarly impactful.
Strengths and limitations of this study

- This study is based on rigorous, multidisciplinary formative research on water insecurity by anthropologists, geographers, nutritionists, statisticians and epidemiologists, among others.

- Data on household water insecurity experiences are being collected in 28 sites across four continents by local partners in widely varying ecological and cultural settings.

- Analytic methods from both classical test and item response theories will be used to develop and evaluate the eventual scale.

- The Household Water Insecurity Experiences Scale will be validated for assessing water insecurity in low-income and middle-income countries. Additional scale assessments necessary for validation in high-income countries are planned.

\section{INTRODUCTION}

Water insecurity, the inability to 'access and benefit from affordable, adequate, reliable and safe water for well being and a healthy life, ${ }^{1}$ has manifold adverse effects on physical $^{23}$ and psychosocial health ${ }^{45}$; undermines productivity ${ }^{6}$; triggers and perpetuates domestic, social and political tensions and conflicts $^{7}{ }^{8}$; and reinforces environmental and social inequities. ${ }^{9}$ Water insecurity has been shown to co-occur with food insecurity, malnutrition and communicable diseases and to produce syndemics, or systemically exacerbating epidemics, ${ }^{10}{ }^{11}$ much like food insecurity and HIV. ${ }^{12}$ Furthermore, water insecurity is projected to worsen in many regions due to climate change and increased inequalities in resource distribution. ${ }^{9}$ 
However, we do not know how many households or individuals globally are affected by water insecurity. Estimates of available surface water derived from satellite imagery suggest that 4 billion people worldwide experience severe water scarcity for at least 1 month of every year, ${ }^{13}$ and this is likely an underestimation given issues with infrastructure and access. Additionally, chronic flooding ${ }^{14}$ and poor water quality ${ }^{15}$ mean that many more individuals are experiencing water insecurity. Currently, measures of water at the national, regional and community levels are used and are referred to as indicators of water scarcity, water poverty or water security. ${ }^{16-19}$ These measures do not capture the range and granularity of how households experience water insecurity, including factors such as perceptions of quality, ${ }^{20}$ instances of water excess $^{21}$ or perceived consequences for psychosocial ${ }^{45}$ and physical health and well-being. ${ }^{22}$ Furthermore, while household-level scales to measure water insecurity have been developed for several sites, for example, in the USA, ${ }^{23}$ Bolivia, ${ }^{4}$ Uganda, ${ }^{24}$ Ethiopia ${ }^{5}$ and Kenya, ${ }^{10}$ their comparability, comprehensiveness and applicability to other sites have not been systematically investigated or validated.

As such, a comprehensive, validated scale to measure the experiences of household or individual water insecurity would enable researchers, practitioners and policymakers to: improve estimates of water insecurity prevalence, identify factors that shape this phenomenon, recognise direct consequences of water insecurity, understand how to more effectively distribute resources, evaluate the impacts and cost-effectiveness of interventions and monitor progress towards the Sustainable Development Goals. ${ }^{25}$ Indeed, in March 2018, the UN's HighLevel Panel on Water cited lack of data on water in many parts of the world as a major challenge, and the need for better data on water as one of nine priority actions. ${ }^{26}$ Given that measures of household food insecurity (eg, Latin American and Caribbean Food Security Scale, ${ }^{27}$ Household Food Insecurity Access Scale, ${ }^{28}$ Food Insecurity Experience Scale ${ }^{29}$ ) have proven vital to implementation and evaluation of policy and programmes, ${ }^{30-32}$ development of an analogous household water insecurity scale is overdue and urgently needed, particularly for assessing water insecurity in low-income and middle-income settings where household water problems tend to be most pronounced and frequent.

Therefore, our objective is to develop and validate the first household water insecurity scale with broad applicability across low- and middle-income settings. The Household Water Insecurity Experiences (HWISE, pronounced H-wise) Research Coordination Network (RCN) was formed to facilitate the multicountry, collaborative data collection process required to validate the planned tool ('the HWISE Scale'). The HWISE RCN brings together a large team of anthropologists, geographers, public health practitioners, physicians, epidemiologists, statisticians, sociologists, nutritionists, inter alia, all of whom have experience with water insecurity, food insecurity and/or scale development across a wide array of settings (http:// www.hwise.org).

\section{METHODS AND ANALYSIS}

Phase 1: item development

Domain specification

Specifying the domains for a scale is the first step in item development (table 1, 1.1). ${ }^{33}$ The boundary of the domain of water insecurity, that is, the underlying construct that the scale will attempt to measure, was based on extensive literature review ${ }^{1}$ and draws on the team's expertise in water insecurity, for example. ${ }^{4} 82335$ We define water insecurity as the condition where 'affordability, reliability, adequacy, and safety [of water] is significantly reduced or unattainable so as to threaten or jeopardize well-being'. ${ }^{1}$

A best practice is to clearly articulate subdomains of the eventual scale, if they are known. ${ }^{34}{ }^{36}$ Although some subdomains of water insecurity have been proposed, ${ }^{151137}$ there is currently no consensus in the literature. Therefore, we will assess subdomains during the analytic phase.

\section{Item generation}

Candidate scale items were identified deductively, based on an extensive literature review of items used in prior site-specific household water insecurity scales ${ }^{1}$ (table 1 , 1.2). This includes team members' prior work in colonias in the USA-Mexico boderlands ${ }^{23}$; a squatter settlement in Cochabamba, Bolivia ${ }^{4}$; in rural, periurban and urban households in Kenya ${ }^{10}$; and elsewhere, including rural areas in Ethiopia ${ }^{5}$ and Uganda. ${ }^{24}$ Initial items include experiences of water insecurity that have consequences for psychosocial and physical health, nutrition, impacts on livelihoods and household economy, and agriculture (online supplementary file 1).

Each question is phrased to elicit experiences within the prior 4 weeks or month (ie, 'In the last fourweeks, how frequently have...'). This recall period was systematically determined using the Delphi method of consensus building with international and local experts in water insecurity, food insecurity and scale development and by comparing the responses in this recall period to a prospective daily recall of water insecurity experiences. ${ }^{10}$ Items were ordered by what we expected to be increasing severity of water insecurity across access, reliability, adequacy and safety. Response options are 'never' (0 times), 'rarely' (1-2 times), 'sometimes' (3-10 times), 'often' (11-20 times), 'always' (more than 20 times), 'not applicable', 'don't know' or refused. Response intervals were also determined using the Delphi method. ${ }^{10}$

The initial set of 32 items is referred to as 'Module Version 1'. This set of items was modified slightly in August 2017 (see 'Mid-study Evaluations' under Phase 3) based on feedback received from consortium members, survey implementers and other water security experts during a 3-day conference at Northwestern University. Modifications included slight rephrasing of 18 items to improve 
Table 1 Overview of planned methods and analyses for the development of the HWISE Scale*

\section{Scale development \\ activity \\ Procedures}

Phase 1: item development
1.1 Domain
Literature review.

specification

1.2 Item generation Literature review and Delphi methodology.

1.3 Content validity By target population: two styles of cognitive interviews were used in the first eight sites, building on Delphi methodology.

1.4 Face validity Pretesting and debriefing with enumerators at each site.

Phase 2: scale development

2.1 Data collection Enumerator training and survey implementation.

2.2 Item reduction We will drop items with cumulative missing cases $>30 \%$ (ie, 'don’t know', 'non-applicable' or true missing responses) in any one site.

We will assess the performance of each item's variation with other items in the scale using a correlation matrix; items with very low $(<0.30)$ interitem correlation coefficients and very low $(<0.30)$ item-total correlation coefficients across multiple sites will be considered for deletion, as will items that misfit the model, that is, with residual correlations $>0.20$.

Item reduction in Rasch paradigm: item severity and item discrimination test.

2.3 Identify factor We will use factor analysis across multiple sites to test for factor structure; items with very low factor structure loadings $(<0.30)$, split factor loadings (high factor loadings $(>0.50)$ in two domains) and high residual variances will be considered for deletion.

2.4 Assess We will use multigroup confirmatory analysis (a form of measurement invariance) on data from measurement multiple sites to test for exact invariance in the hypothetical scale; invariance will be assessed in equivalence terms of factor structure (configural model), factor loadings (matric model), mean intercepts (scalar model) and factor means (strict model).

We will use confirmatory factor analysis alignment optimisation to estimate the group-specific factor means and variances of scale items across all sites; it assesses approximate invariance of scale items across multiple sites.

Phase 3: scale evaluation

3.1 Score scale items Finalised scale items will be used in their unweighted form as sum scores or in weighted form as factor scores.

3.2 Assess reliability We will use Cronbach's alpha and the Rasch reliability statistic to test the internal consistency of the (internal consistency) scale items within each site and aggregated across sites.

of scale items

3.3 Assess scale validity

We will measure predictive, convergent and discriminant validity of the final scale items using criteria that were selected based on their strong theoretical relevance in the water insecurity literature; tests of water insecurity differences between 'known groups' will also be performed.

${ }^{*}$ Adapted from ref ${ }^{34}$.

comprehension by participants and to elicit experiences related to water overabundance, two questions were added in an effort to capture cultural components of water and six items were eliminated for being too rare or idiosyncratic. The resultant set of 28 items is referred to as 'Module Version 2' (online supplementary file 1).

Content validity

Content validity (ie, if items adequately measure the domain of interest; table 1, 1.3) was assessed in the first eight sites through cognitive interviews with 12 purposively selected individuals. Participants were asked to 'think aloud' or 'tell [the enumerator] about' their understanding of each of the water insecurity items as they completed the pilot survey. Interviewers recorded any issues and probed in detail on each as participants responded to the items. ${ }^{38}$ This process built on the Delphi methodology used to develop the Kenya-specific scale. ${ }^{10}$

\section{Face validity}

Face validity, also part of item development, is assessed at each site (table 1, 1.4). First, the survey is translated from English into the language(s) of implementation and then back-translated. Then, enumerators, the predominance of whom are recruited from the target population, pretest surveys with one another to ensure that questions are appropriate to the setting, that the concept of water insecurity is understood and that translations are consistent with local dialects, that is, that they are linguistically and culturally appropriate translations. ${ }^{29}$ 
Site leads debrief enumerators after each day of data collection and record all the details as project field notes to further ensure face validity. Debriefs are centred on experiences in the community, survey questions that are difficult to administer and any other problems encountered. At the end of data collection for the site, enumerators engage in a final debrief, and in some cases, use a semistructured survey that pulls the same information from across the entire site. Site leads are also interviewed at the end of study activities by members of the HWISE RCN regarding their experiences with project implementation, perceptions of questions by enumerators and participants and any additional topics that should be included or excluded in the final survey. These debriefing interviews with site leads will provide additional feedback to iteratively improve training and item refinement.

\section{PHASE 2: SCALE DEVELOPMENT Data collection \\ Sites}

Cross-sectional surveys were initially planned for six sites that would leverage investigators' active research: Bangladesh, Brazil, Guatemala, Kenya, Nepal and Tajikistan; that is, they were selected out of convenience. Subsequently, 22 more were added because additional sites would allow us to test the instrument across more heterogeneous cultural and geographic settings (figure 1), permit an iterative analysis of the instrument (compared with 'Mid-study evaluation') and make a number of statistical analyses possible (table 2) ${ }^{39}$ These additional 22 sites were added by soliciting collaborators from professional networks across academic institutions as well as non-governmental and governmental organisations using convenience sampling. In selecting sites, we sought maximal heterogeneity in region of the world, infrastructure (eg, urban and rural, formal and informal settlements) and problems with water (eg, flooding, drought, chronic scarcity and intermittent supplies). We also considered cost and feasibility of timely implementation.

\section{Participant selection}

To participate, individuals must be 16 or 18 years of age or older (depending on age of consent at each site), identify themselves to the interviewer as being knowledgeable about water acquisition and use within their households and consent to participate. Participants are not remunerated for participation in the survey.

The target sample size at each site is 250 individuals. We consider this sample size as the minimum needed for assessing the magnitude of correlation between the observed variables and associated factor(s) and obtaining a sample pattern that is stable and approximates the population pattern. ${ }^{40}$ If sites cannot achieve the target sample size, variation of estimated statistics will be reviewed to determine if the data can be included in the final validation of the scale.

The preferred sampling strategy for the study is random sampling of mutually exclusive and exhaustive categories of participants in areas of known high, moderate and low

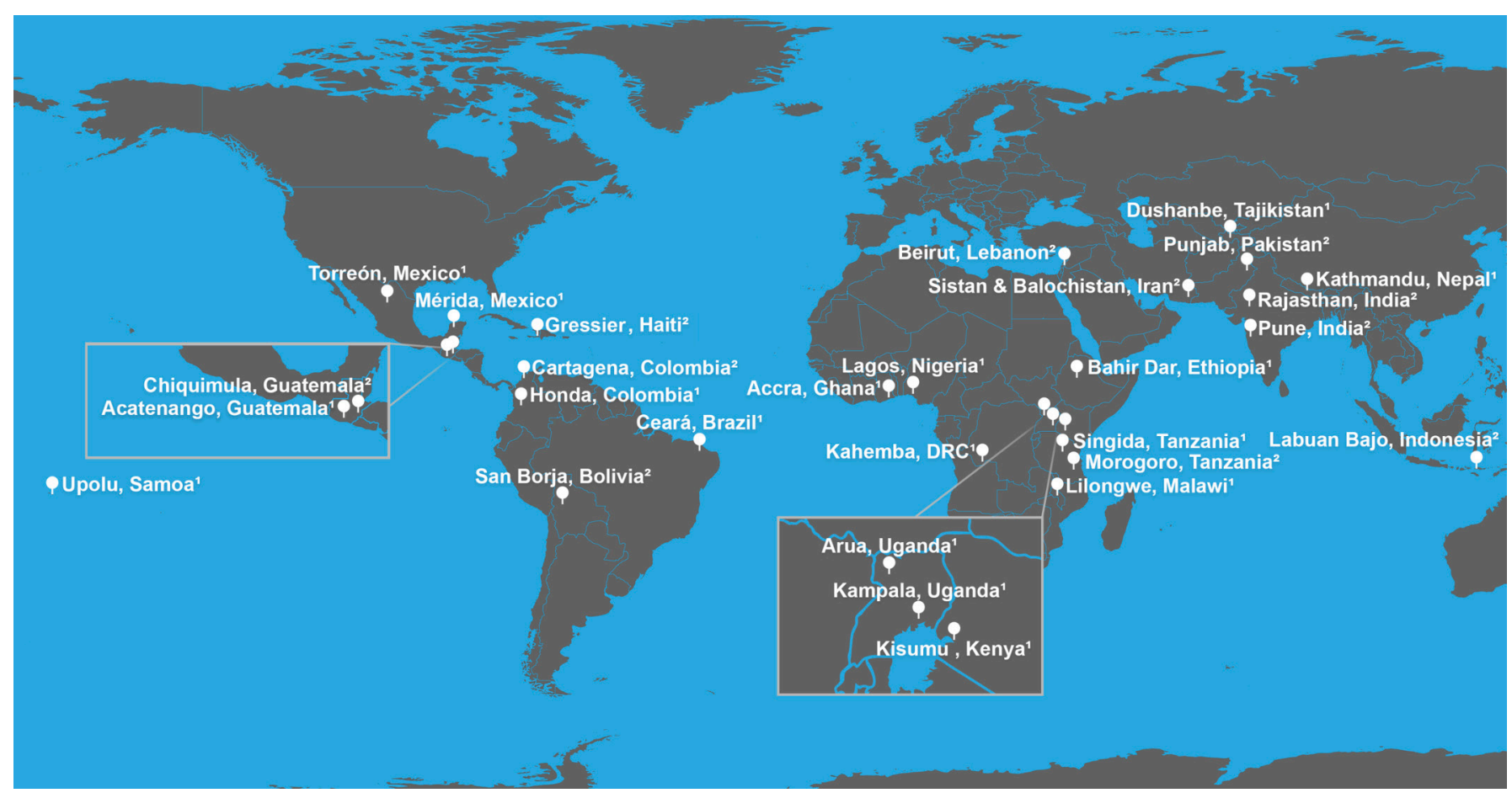

Figure 1 Map of HWISE study sites. ${ }^{1}$ Sites using Module Version $1 ;{ }^{2}$ Sites using Module Version 2. Image credit: Frank Elavsky, Northwestern University Information Technology, Research Computing Services. HWISE, Household Water Insecurity Experiences. 


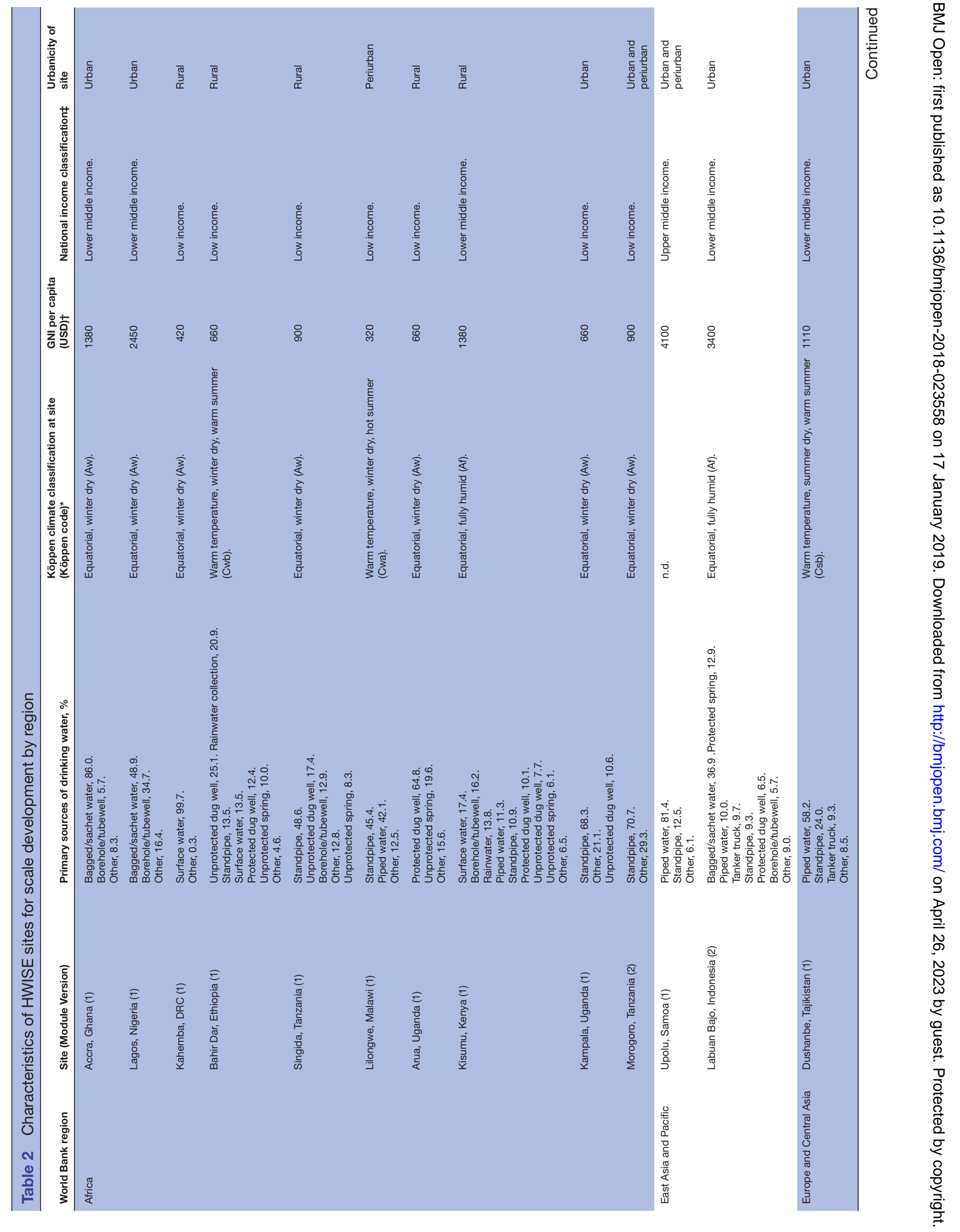




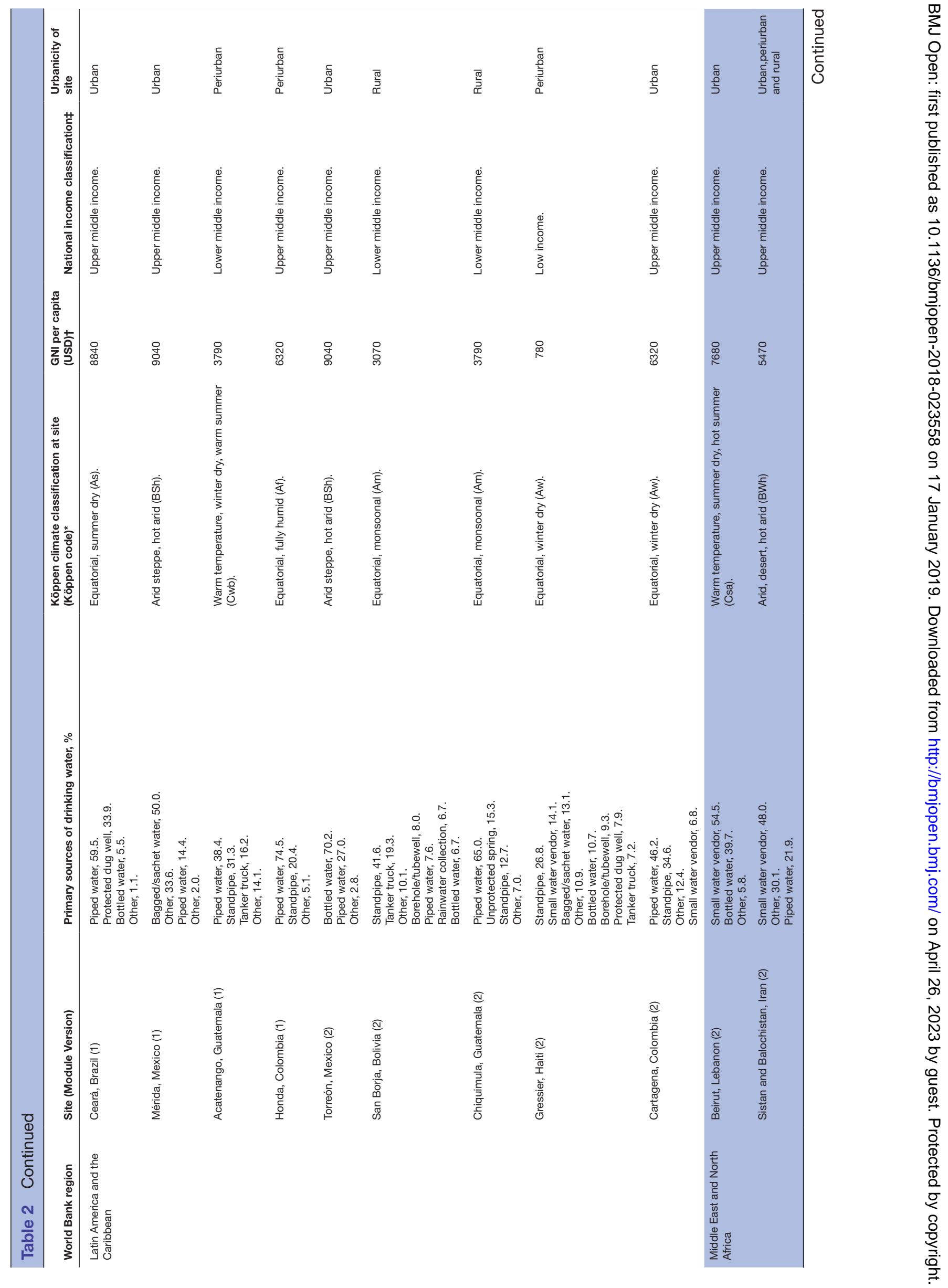


water insecurity. In standalone HWISE sites, participant selection follows a simple randomised or cluster-randomised sampling strategy (table 3). In several sites, however, the HWISE survey is administered as part of a larger ongoing project with a predetermined survey design (eg, in Singida, Tanzania: NCT02761876; Kahemba, Democratic Republic of Congo: NCT03157336), such that simple random sampling is not possible.

Sites with simple randomised sampling employ a random-walk sampling method. ${ }^{41}$ With the simple randomised sampling strategy, a random number generator (eg, dice or random number generating application) with set parameters (ie, less than 20, less than 30 and so on) determines which households to survey (and, if needed, the direction of the random walk). Surveys are administered to each household corresponding to the random number, such that a random draw of the number 3 indicates that every third household should be sampled. For sites using a cluster-randomised sampling strategy, the region is first mapped using a grid or satellite imagery (eg, Google Maps) to identify population density based on the number of habitable structures. Clusters are selected from this grid, and households within clusters are randomly sampled in proportion to structure or population density using a random number generator, similar to the simple randomised sampling strategy. Cluster randomisation is preferred, but simple random sampling is used when cluster data are not available, typically in sparsely populated settings.

\section{Participant involvement}

Although formative work drew on ethnographic research that included participant involvement and the idea to develop this scale came from experiences with participants in Kenya, ${ }^{1}$ no participants were involved in developing the actual protocol. Participant involvement (eg, cognitive interviewing; table 1, 1.3) began with refinement of survey items once the initial list was created. Participants were not involved in developing plans for the design or implementation of the study, and participants will not be involved in the interpretation of results or write-up of the manuscript. Although identifiable data were not collected in most sites, we plan to summarise our findings in site-specific summary reports that site investigators will disseminate to communities in which the data were collected. The final scale and other findings will be made available via open-access publication and be publicised through public relations and media outreach at our respective institutions.

\section{Training}

An HWISE training manual was developed to provide guidance on implementation. ${ }^{42}$ This manual outlines preferred sampling strategy, minimum sample size, instructions for collecting data and choosing unique participant identification numbers and detailed information explaining the rationale for each water insecurity 


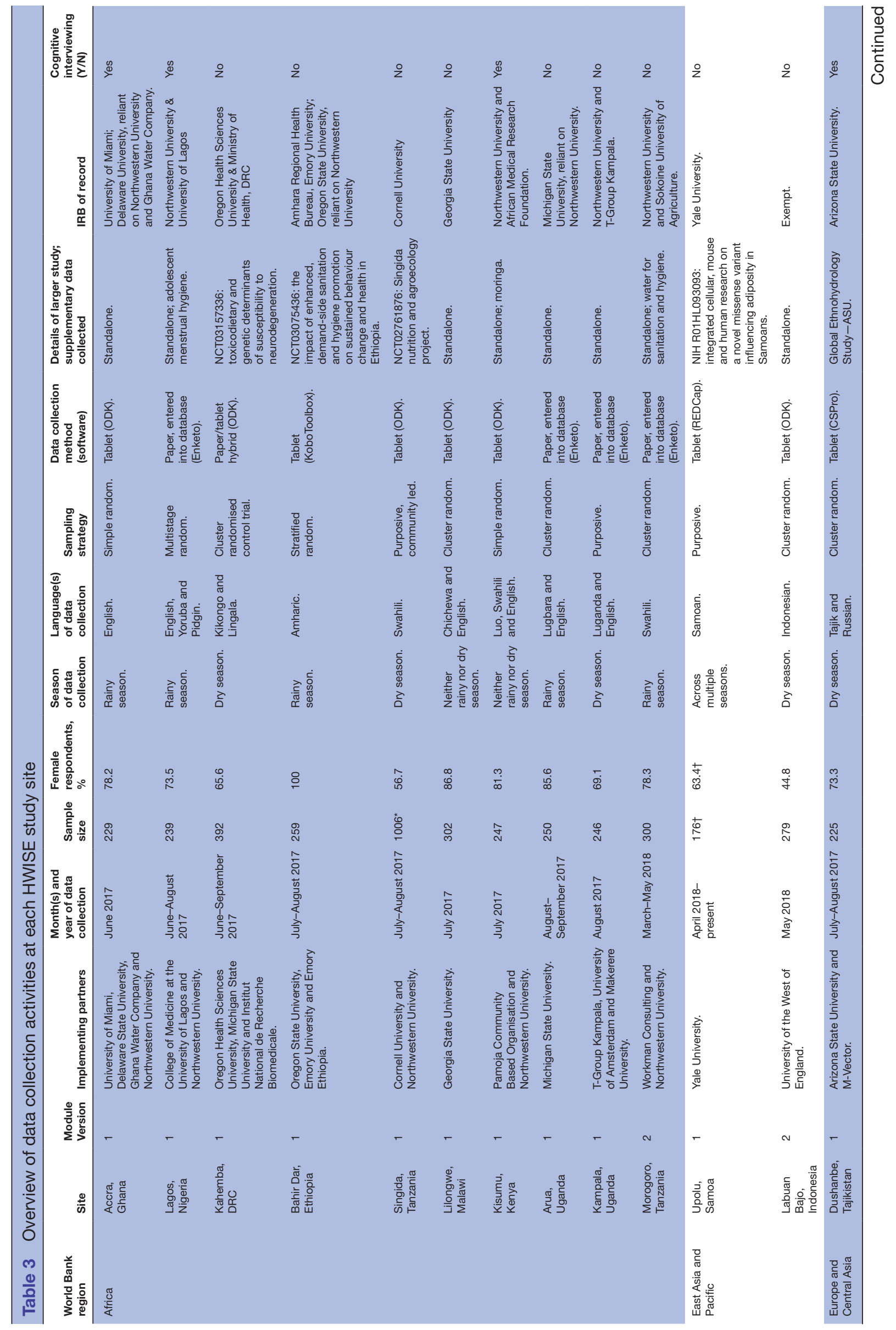

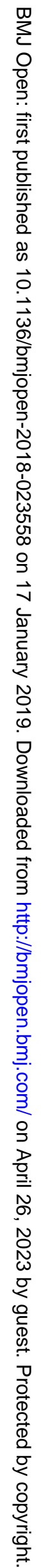




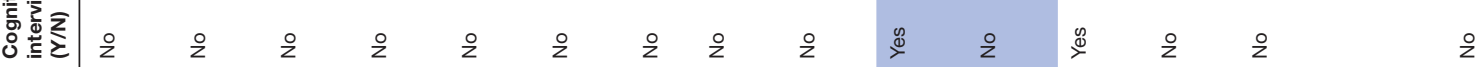

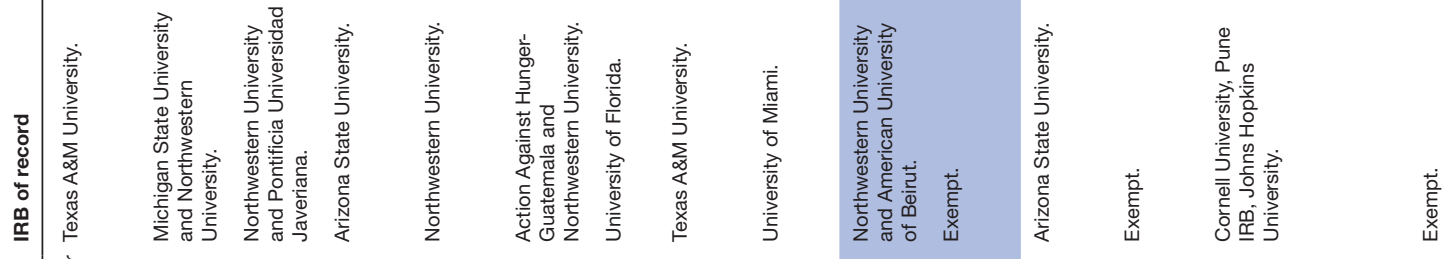

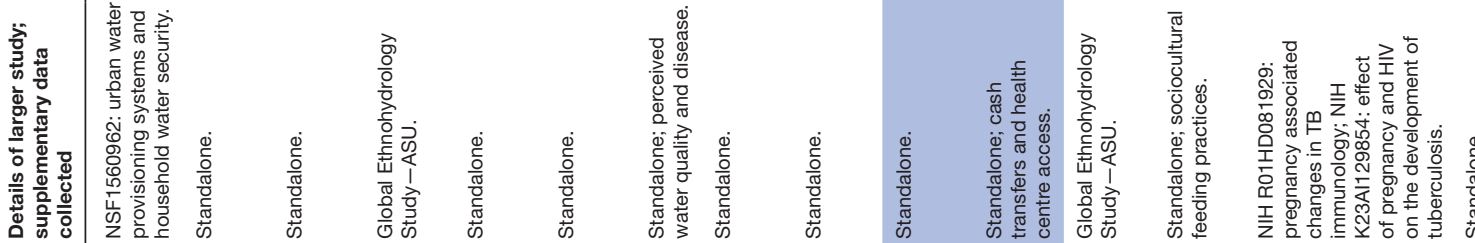

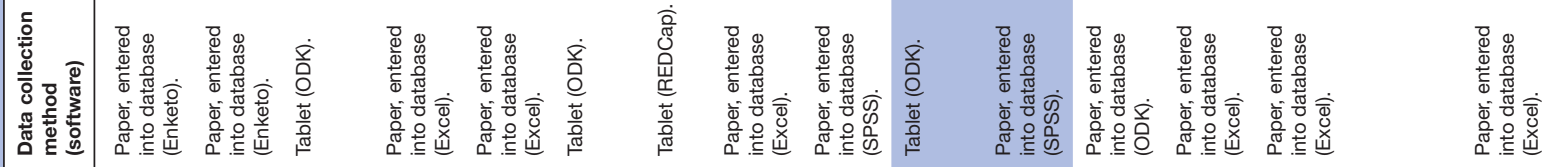

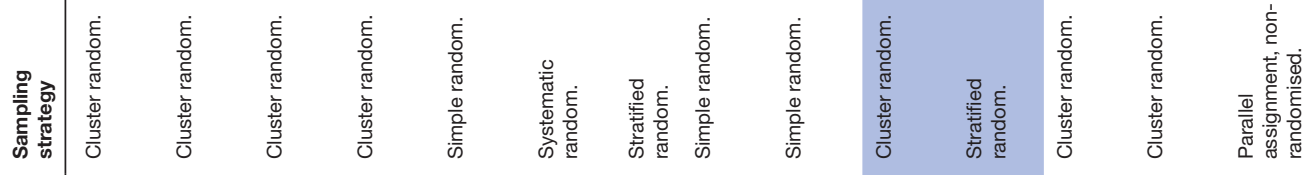

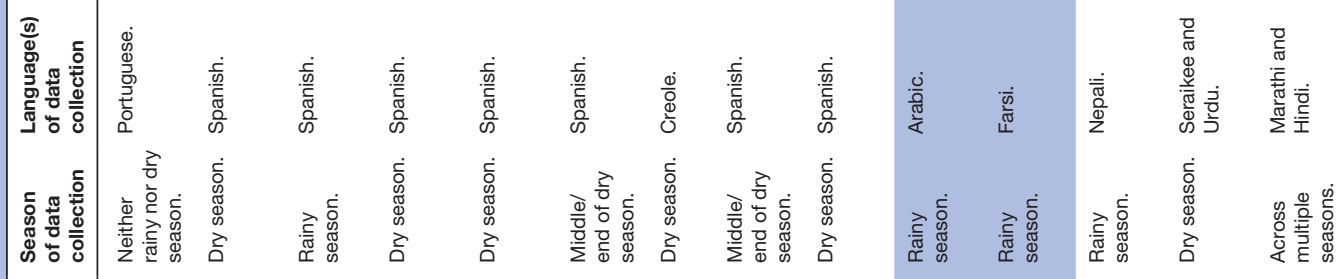
$\frac{1}{2}$

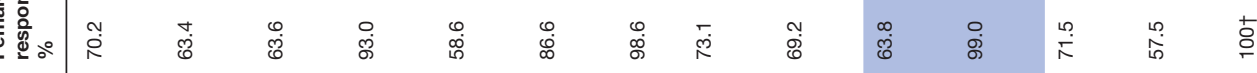

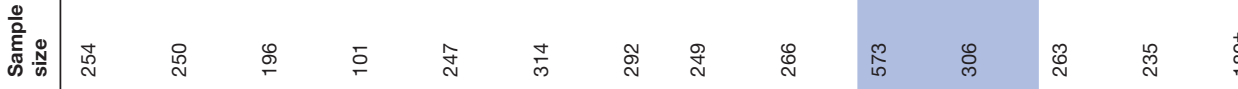

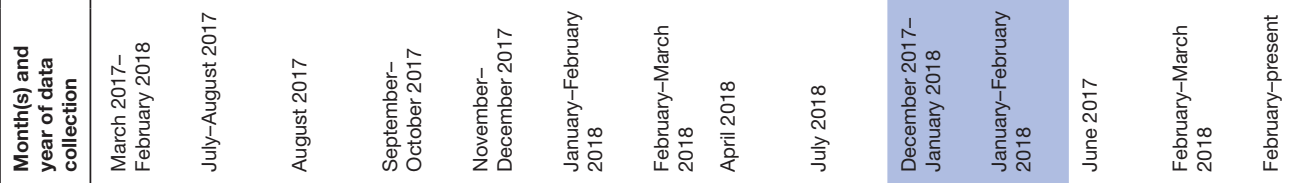

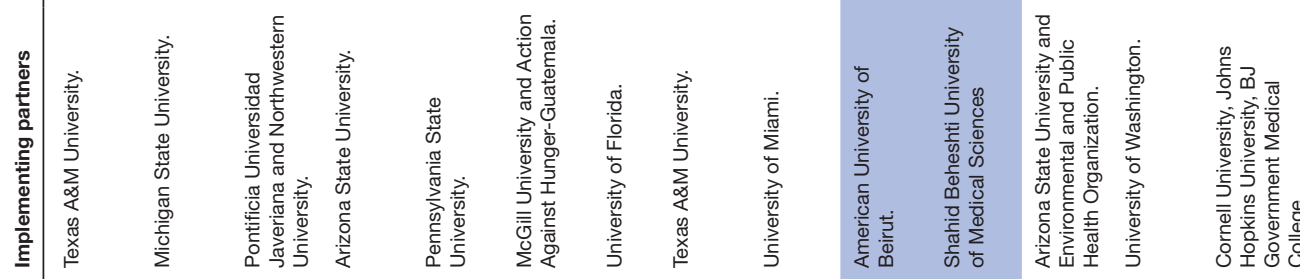

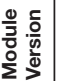


item and survey section (online supplementary material 1). This manual has been translated from English into Arabic and adapted for use in Uganda.

Each site has at least one formally appointed lead investigator responsible for consistent training, sampling, recruitment and data collection. In each site, 5-10 enumerators with survey implementation experience, knowledge of the area and context, and fluency in the local language(s) are recruited. Enumerators at all sites attend a 1-2 day training session. The first portion of the training curriculum is didactic and follows the survey manual. The rest of the training is interactive and tactile, with enumerators piloting the survey with one another and troubleshooting any issues that arise. After the initial training, the site lead and/or study coordinator accompany enumerators during data collection and provide feedback until enumerators are sufficiently comfortable with the survey to administer it with minimal guidance.

\section{Data collection and management}

After consent, enumerators conduct interviews with the person who identifies themselves to the enumerator as being knowledgeable about water acquisition and use in his or her household. In addition to the water insecurity experience items described above (Module Versions 1 or 2), data are collected on sociodemographic characteristics; water acquisition, use and storage; household food insecurity (using the Household Food Insecurity Access Scale ${ }^{28}$ ); perceived stress (using a modified, four-item perceived stress scale ${ }^{43}$ ); and data quality (online supplementary material 2 ). These additional data will be used to validate the scale and explore other water insecurity phenomena in a cross-cultural manner. ${ }^{44}$ Each interview lasts approximately 45 minutes, and we expect data collection to last approximately 10-14 days in each standalone survey site (table 3 ).

Implementation of HWISE data collection began in March 2017 and is expected to end in late 2018. Data collection with Module Version 1 began in March 2017 and is ongoing (table 3 , currently $\mathrm{n}=4817$ ). Data collection using Module Version 2 began in November 2017 and is also ongoing (currently $n=3310$ ).

Data are collected using both paper and tablet-based collection platforms, that is, Open Data Kit (ODK), opendatakit.org $^{45}$; CSPro, csprousers.org; and KOBOToolbox (Cambridge, Massachusetts, USA; kobotoolbox.org). To reduce data collection errors, tablet-based platforms are programmed to include permissible ranges of responses, skips for questions that are not applicable and survey items in the language(s) most common to each study site. Most responses from paper surveys are entered by enumerators, study coordinators, data managers and/or site primary investigators into an online data collection platform (Enketo; enketo.org). Microsoft Excel is used when reliable internet access is unavailable.

Data are uploaded to a secure centralised aggregate server (Google App Engine). Stata 14 is used for data cleaning following a data cleaning protocol agreed on by the HWISE RCN (online supplementary material 3).

\section{Implementation fidelity}

To ensure implementation fidelity, enumerators are debriefed daily following data collection. Both enumerators and site PIs are debriefed postimplementation (online supplementary material 4). Furthermore, each survey contains a module on perceived data quality (eg, explanation of missing data, distractions and issues with recruitment) that is filled in by the enumerator immediately postinterview.

\section{Analytic strategy}

Three software packages will be used for analyses: Stata 14 to run basic descriptive statistics; Mplus version 8 (Muthén \& Muthén, Los Angeles, California, USA) and Stata 14 for classical test theory analysis; and WINSTEPS (Winsteps, Beaverton, Oregon, USA) for item response theory (Rasch) analysis.

Scale development (table 1, 2.1-2.4) and evaluation (table 1, 3.1-3.3) will be informed by analyses corresponding to two scaling theories: classical test theory, ${ }^{46}$ implemented by factor analysis, and item response theory, ${ }^{47}$ using Rasch models.

\section{Item reduction}

First, items with large cumulative missing cases (>30\%), that is, 'don't know', 'non-applicable' or true missing responses, will be dropped (table 1, 2.2). This will help to eliminate items that are not understood or are not widely applicable, and therefore do not reflect cross-cultural experiences of water insecurity.

Thereafter, items will be further dropped based on low correlation coefficients. In classical test theory, we will identify items with low $(<0.30)$ interitem and item-total correlation coefficients across the multiple sites in this study. ${ }^{1048}$

Within the Rasch paradigm, we will identify and remove items that misfit the models by assessing infit and outfit. ${ }^{49} 50$ Conditional item independence (ie, items conditional on the scale that are not correlated) will be assessed using residual correlation metrics. Items will be dropped if residual correlation is $>0.20{ }^{51}$

\section{Identify factor structure}

Factor analysis with data from multiple sites will be used to identify the optimal latent structure (table 1, 2.3). We will examine this structure for each site, comparing factor structures, magnitudes of factor loadings, eigenvalues for sample correlation matrices and global model fitness statistics. Items with low factor loadings $(<0.30)$, split factor loadings and high residual variances $(>0.50)$ will be considered for deletion. $^{34}$

\section{Assess measurement equivalence}

Measurement equivalence concerns the extent to which the psychometric properties of the observed indicators are generalisable across groups or over time. ${ }^{52-55}$ It holds 'when a test measures a construct in the same way regardless of group membership and is violated when individuals from 
different groups respond to the test in a dissimilar manner'. 39 A violation of equivalence implies our inability to make comparisons about the measurement and meaning of scale values across groups (eg, sites, cultures and languages). ${ }^{39}$ To determine measurement equivalence across sites using Module Versions 1 and 2, we will use multigroup confirmatory factor analysis and alignment optimisation. ${ }^{56-58}$

\section{PHASE 3: SCALE EVALUATION}

\section{Score scale items}

Once a water insecurity scale that is equivalent across sites is provisionally identified, we will use scale scores in both weighted forms (factor scores) and unweighted forms (sum scores) to assess the external validity of our scale.

\section{Reliability}

To test for the reliability (internal consistency) of the items, we will estimate Cronbach's alpha for both site-specific and aggregate-level data. ${ }^{59}$ The Rasch reliability statistic is analogous to Cronbach's alpha. In our analyses, we will consider reliability to be ideal if it is greater than $0.80 .^{59}$

\section{Validity}

We will examine three types of validity: predictive, convergent and discriminant validity. Predictive validity is 'the extent to which a measure predicts the answers to some other question or a result which it ought to be related with'. ${ }^{60}$ Using both linear regression and structural equation models, we will test for predictive validity by regressing HWISE Scale scores on eg, food insecurity, perceived stress and income.

Convergent validity is the 'degree to which scores on a studied instrument are related to measures of other constructs that can be expected on theoretical grounds and accumulated knowledge to be close to the one tapped into by this instrument'. ${ }^{48}$ To test for convergent validity, we will assess the relationships between HWISE Scale scores and individual items that have shown to be closely related to the concept of water insecurity. Specifically, we will use linear regression to examine the strength of the relationships between HWISE Scale scores and eg, time to water source, number of trips to water source and amount of money spent purchasing water. Larger correlation and regression coefficients and smaller SD of residuals will be indicative of support for convergent validity.

Discriminant validity is the "degree to which scores on a studied instrument are differentiated from behavioral manifestations of other constructs'. ${ }^{48}$ A test of differentiation between 'known groups' will be conducted using the Student's $t$-test ${ }^{34} 48$; these groups will be based on accumulated knowledge. We will determine the distribution of household water insecurity scores across known groups, eg primary source of drinking water (improved vs unimproved sources), water treatment (treated vs untreated), gender of household head (male vs female) and injuries associated with water acquisition (yes vs no). ${ }^{45102461}$ Under the Rasch measurement model, differentiating between known groups will also be conducted using differential item functioning. We will determine whether each scale item performs differently in each of the subgroups. Differential item functioning is attained when the probabilities of an item being endorsed is unequal for the two subgroups. ${ }^{1062}$

In sum, selection of the set of items to be included in the final scale will be based on several criteria. The criteria for inclusion of an item are: reliable in each site, fits theoretically and empirically with concepts related to water insecurity, has face and content validity in each site, shows equivalent measurement and meaning across sites and contributes to predictive, convergent and discriminant validity in each site. ${ }^{63}$ We anticipate that not every item will meet each criterion perfectly, and judgement about tradeoffs of which items to include will be required. These judgements will be made considering the additional criteria of having a diversity of items in the final scale that cover as many facets of water insecurity as reasonably possible. We anticipate that the final scale will have fewer than 20 items, which will reduce the likelihood of participant fatigue and make its widespread application more feasible.

\section{Midstudy evaluations}

In August 2017, 5 months after data collection began in 8 of 16 Module Version 1 sites, HWISE RCN members met at Northwestern University to review and discuss data received to date and thematically sort HWISE items. This led to the reduction and refinement of HWISE for the second wave of survey implementation (Module Version 2), which is being administered across 12 sites (table 2 and 3). In February 2018, HWISE RCN members involved in scale validation met at McGill University to review Module Version 2 responses to date and further refine the survey. Members of the analytic team also hold regular conference calls to review subsequent results and complete the scale validation process.

\section{ETHICS AND DISSEMINATION}

All participants are verbally consented by enumerators in their language of choice using a standardised script (online supplementary material 2). Study activities are reviewed and approved by all appropriate ethical review boards (table 3 ).

\section{Author affiliations}

${ }^{1}$ Department of Anthropology, Institute for Policy Research, Northwestern University, Evanston, Illinois, USA

${ }^{2}$ Department of Anthropology, Northwestern University, Evanston, Illinois, USA ${ }^{3}$ Department of Medicine, University of California, San Francisco, California, USA ${ }^{4}$ Center for Research on Population and Health, American University of Beirut, Beirut, Lebanon 
${ }^{5}$ School of Human Evolution and Social Change, Arizona State University, Tempe, Arizona, USA

${ }^{6}$ Department of Health Promotion, Education, and Behavior, University of South Carolina, Columbia, South Carolina, USA

${ }^{7}$ Department of Geography, Texas A\&M University, College Station, Texas, USA ${ }^{8}$ Institute for Global Food Security, McGill University, Montreal, Québec, USA

${ }^{9}$ Department of Geography, University of Miami, Coral Gables, Florida, USA

Acknowledgements We are very grateful to our participants, without whom this scale would not be possible. We would also like to warmly and sincerely thank the field teams for their hard work and dedication to this project: Velly Emina, Victoria Yesufu, Annah Adakhilan, Adekunbi Adejokun, Adebari Adewunmi, Damola Adelakun, Nike Odunaike, Anthony Sekoni, Ramon Babamole and Kayode Badru (Lagos, Nigeria); Prashant Rimal, Sarita Lawaju, Roshna Twanabasu, Renuka Baidhya, Ayaswori Byanju, Menuka Prajapati and Ranju Magar (Kathmandu, Nepal); Andrew Mvula, Wisdom Mwale, Faith Kanyika, Wyson Samata, Fanney Kanyenda and Mcdonad Mpangwe (Lilongwe, Malawi); Maxwell Akosah-Kusi, David Okai Nunoo, Rita Antanah and Michael Nyoagbe (Accra, Ghana); Gulnoza Sharipova, Ganjina Hudoieva, Gulsara Nozirova, Shahobiddin Murodov, Navrasta Shoeva, Nasiba Gadoeva, Markhabo Ibragimova and Vahidova Saodat (Dushanbe, Tajikistan); Milton Marin Morales (San Borja, Bolivia); Wicklife Odhiambo Orero, Judith Atieno Owuour, Philip Otieno Orude, Sylvia Achieng Odhiambo and Kennedy Oduor (Kisumu, Kenya); Daniel Guerrero, Daniela Avila, Kelly Johana Diaz Ceballos, Valentina Giraldo Bohorquez and Pedro Castillo (Honda, Colombia); Moses Mwebaza, Dorren Bamanya, Alines Mpandu, Alex Kazooza, John Ssemwogerer, Olivia Nakamya, Kimbugwe Muhammed, Simon Kyagera, Gerald Ssozi, Solomon Wakida, Matteo Andrea Corsini, Ann Apio, Atim Catu and Nahwera Julie (Kampala, Uganda); Alonzi Francis, Candia Alex, Alesi Christine, Adjonye Doreen, Aputru Florence and Ayakaka Beatrice (Arua, Uganda); Michel Lupamba, Mary Aziza Mulumba, Smith Tshibulenu, Kevis Kamanda and Thérèse Hosa (Kahemba, Democratic Republic of Congo); Eliwaza Mpeko, James Raphael, Emmanuel Katabi, Raziki Amon, Theresia Ononga, Eliofoo Yohana, Neilu Issack, Faudhia Kitiku, Janeth Kacholi, Nyambuli Deus, Oliver Mwanjati, Faith Titus, Fadhali Nyasiro and Mwantum Mkama (Singida, Tanzania); Robinson Bernier, Berlyne Bien-Aimé and Claude Civil (Gressier \& Léogâne, Haiti); Luambano Kihoma, Generoza Amos, Patricia Msolla, Peter Amandus, Cyril Lissu, Fredy Bernard, Joylight Mbitta, Raphael Chelele, Alan Kimbita and Elizabeth Msiuike (Morogoro, Tanzania); Daniel Eduardo Lemaitre, Saray Noel Tarra, Luis Murillo Ortega, Natalia Yepes Montes, Jairo Andres Aviles Rojano, Juan Jose de la Espriella Correa, Marcela Florez, Juan Andres Barrios, Stephanie Escobar Diaz, Yuriza Martinez and Carlos Anibal Batista Ruiz (Cartagena, Colombia); Desetaw Asnakew, Roza Abesha, Tigist Abebe and Yeserash Gashu (Bahir Dar, Ethiopia). Furthermore, we are grateful to Northwestern University Information Technology's Research Computing Services team, especially Frank Elavsky for his creation of figure 1.

Collaborators HWISE Research Coordination Network: Ellis Adams; Farooq Ahmed; Mallika Alexander; Mobolanle Balogun; Michael Boivin; Genny Carrillo; Kelly Chapman; Stroma Cole; Hassan Eini-Zinab; Jorge Escobar-Vargas; Matthew C. Freeman; Hala Ghattas; Ashley Hagaman; Nicola Hawley; Kenneth Maes; Jyoti Mathad; Patrick Mbullo Owour; Javier Moran; Nasrin Omidvar; Amber Pearson; Asher Rosinger; Luisa Samayoa-Figueroa; Ernesto Sánchez-Rodriguez; Jader Santos; Marianne V. Santoso; Sonali Srivastava; Chad Staddon; Andrea Sullivan; Yihenew Tesfaye; Nathaly Triviño-León; Alex Trowell; Desire Tshala-Katumbay; Raymond Tutu; Felipe Uribe-Salas; Elizabeth Wood; and Cassandra Workman.

Contributors SLY conceptualised the study, developed HWISE items, wrote the manuscript, obtained funding and oversaw data collection and analysis. SMC helped develop HWISE items, wrote the manuscript, prepared the field manual and managed data. GOB developed the data analysis and validation plan and helped write the analytic section of the manuscript. TBN assisted with study design and supported scale analysis and validation. ZJ proposed data analysis and helped write the analytic section of the manuscript. JDM developed tools for data collection and managed data. $A A B$ proposed analyses for item development. EAF and $\mathrm{HM}-\mathrm{Q}$ proposed data analysis. WEJ conceptualized the study and developed HWISE items. RCS supported development of HWISE items and assisted with preparation of the manual. JBS proposed analyses for item development. AW conceptualized the study, developed HWISE items and proposed analyses for item development. HWISE RCN members provided substantial contributions to data acquisition and interpretation. All authors critically reviewed and approved the final draft of the manuscript.

Funding We gratefully acknowledge our funders: the Competitive Research Grants to Develop Innovative Methods and Metrics for Agriculture and Nutrition Actions (IMMANA). IMMANA is funded with UK Aid from the UK government. This project was also supported by the Buffett Institute for Global Studies and the Center for Water Research at Northwestern University; Arizona State University's Center for Global Health at the School of Human Evolution and Social Change and Decision Center for a Desert City (National Science Foundation SES-1462086); the Office of the Vice Provost for Research of the University of Miami; the National Institutes of Health grant NIEHS/FIC R01ES019841 for the Kahemba Study, DRC. Texas A\&M University-CONACyt Grant supported data collection in Torreon, Mexico. We also acknowledge the National Science Foundation's HWISE Research Coordination Network (BCS-1759972) for support of the collaboration. SLY was supported by the National Institutes of Health (NIMH R21 MH108444; NIMH K01 MH098902). WEJ was supported by the National Science Foundation (BCS-1560962).

Competing interests None declared.

Patient consent Not required.

Ethics approval Northwestern University, African Medical Research Foundation (AMREF), American University at Beirut, Arizona State University, Cornell University, Delaware State University, Florida State University, Georgia State University, Ghana Water Company, International Centre for Diarrhoeal Disease Research, Bangladesh (icddr,b), Johns Hopkins University, College of Medicine at the University of Lagos, University of Miami, McGill University, Michigan State University, Nepal Health Research Council, Oregon Health Sciences University, Oregon State University, Penn State University, Pontificia Universidad Javeriana, Sokoine University of Agriculture, Texas A\&M University, T-Group Kampala and Yale University.

Provenance and peer review Not commissioned; externally peer reviewed.

Data sharing statement Data are currently being collected and are not yet available for access.

Open access This is an open access article distributed in accordance with the Creative Commons Attribution 4.0 Unported (CC BY 4.0) license, which permits others to copy, redistribute, remix, transform and build upon this work for any purpose, provided the original work is properly cited, a link to the licence is given, and indication of whether changes were made. See: https://creativecommons.org/ licenses/by/4.0/.

\section{REFERENCES}

1. Jepson WE, Wutich A, Colllins SM, et al. Progress in household water insecurity metrics: a cross-disciplinary approach. Wiley Interdisciplinary Reviews: Water 2017;4:e1214-21.

2. Geere JA, Hunter PR, Jagals P. Domestic water carrying and its implications for health: a review and mixed methods pilot study in Limpopo Province, South Africa. Environ Health 2010;9:52-13.

3. Prüss-Ustün A, Bartram J, Clasen T, et al. Burden of disease from inadequate water, sanitation and hygiene in low- and middle-income settings: a retrospective analysis of data from 145 countries. Trop Med Int Health 2014;19:894-905.

4. Wutich A, Ragsdale K. Water insecurity and emotional distress: coping with supply, access, and seasonal variability of water in a Bolivian squatter settlement. Soc Sci Med 2008;67:2116-25.

5. Stevenson EG, Greene LE, Maes KC, et al. Water insecurity in 3 dimensions: an anthropological perspective on water and women's psychosocial distress in Ethiopia. Soc Sci Med 2012;75:392-400.

6. Sorenson SB, Morssink C, Campos PA. Safe access to safe water in low income countries: water fetching in current times. Soc Sci Med 2011;72:1522-6.

7. Mason LR. Examining relationships between household resources and water security in an Urban Philippine community. J Soc Social Work Res 2014;5:489-512.

8. Wutich A, Food BA. Water, and scarcity: toward a broader anthropology of resource insecurity. Current Anthropology 2014;55:444-68.

9. Cook C, Bakker K. Water security: debating an emerging paradigm. Global Environmental Change 2012;22:94-102.

10. Boateng GO, Collins SM, Mbullo P, et al. A novel household water insecurity scale: procedures and psychometric analysis among postpartum women in western Kenya. PLoS One 2018;13:e0198591.

11. Workman CL, Ureksoy $\mathrm{H}$. Water insecurity in a syndemic context: understanding the psycho-emotional stress of water insecurity in Lesotho, Africa. Soc Sci Med 2017;179:52-60.

12. Weiser SD, Young SL, Cohen CR, et al. Conceptual framework for understanding the bidirectional links between food insecurity and HIV/AIDS. Am J Clin Nutr 2011;94:1729S-39.

13. Mekonnen MM, Hoekstra AY. Four billion people facing severe water scarcity. Sci Adv 2016;2:e1500323. 
14. Hirabayashi $\mathrm{Y}$, Mahendran R, Koirala $\mathrm{S}$, et al. Global flood risk under climate change. Nat Clim Chang 2013;3:816-21.

15. Bain R, Cronk R, Hossain R, et al. Global assessment of exposure to faecal contamination through drinking water based on a systematic review. Trop Med Int Health 2014;19:917-27.

16. Falkenmark M, Lundqvist J, Widstrand C. Macro-scale water scarcity requires micro-scale approaches. Aspects of vulnerability in semiarid development. Nat Resour Forum 1989;13:258-67.

17. Rijsberman FR. Water scarcity: Fact or fiction? Agric Water Manag 2006;80:5-22.

18. Sullivan CA, Meigh JR, Giacomello AM, et al. The water poverty index: development and application at the community scale. Nat Resour Forum 2003;27:189-99.

19. Global Water Partnership. Proceedings from the GWP workshop: assessing water security with appropriate indicators. Stockholm, 2014:120.

20. de França Doria M. Factors influencing public perception of drinking water quality. Water Policy 2010;12:1-19.

21. Grey D, Sadoff CW. Sink or Swim? Water security for growth and development. Water Policy 2007;9:545-71.

22. Bartram J, Lewis $\mathrm{K}$, Lenton $\mathrm{R}$, et al. Focusing on improved water and sanitation for health. The Lancet 2005;365:810-2.

23. Jepson W. Measuring 'no-win' waterscapes: Experience-based scales and classification approaches to assess household water security in colonias on the US-Mexico border. Geoforum 2014:51:107-20.

24. Tsai AC, Kakuhikire B, Mushavi R, et al. Population-based study of intra-household gender differences in water insecurity: reliability and validity of a survey instrument for use in rural Uganda. $J$ Water Health 2016;14:280-92.

25. UN General Assembly. Transforming our world: the 2030 Agenda for sustainable development [Internet]. 2015. Report No.: a/RES/70/1 https://sustainabledevelopment.un.org/content/documents/ $21252030 \% 20$ Agenda\%20for\%20Sustainable\%20Development\% 20web.pdf

26. High Level Panel on Water. Making every drop count: an agenda for water action. $2018 \mathrm{https} / /$ sustainabledevelopment.un.org/content/ documents/17825HLPW_Outcome.pdf

27. Pérez-Escamilla R, Melgar-Quiñonez $\mathrm{H}$, Nord M, et al. Escala Latinoamericana y Caribeña de Seguridad Alimentaria (ELCSA) [Latinamerican and Caribbean Food Security Scale]: Perspectivas en Nutrición Humana, 2007:117-34.

28. Coates J, Swindale A, Bilinsky P. Household food insecurity access scale (hfias) for measurement of food access: indicator guide. Washington DC: Food and Nutrition Technical Assistance Project, 2007.

29. Ballard T, Kepple AW, Cafiero C. The food insecurity experience scale. Rome, Italy: Food and Agriculture Organization, 2013.

30. Cafiero C, Viviani S, Nord M. Food security measurement in a global context: the food insecurity experience scale. Measurement 2018;116:146-52.

31. Pérez-Escamilla R. Can experience-based household food security scales help improve food security governance? Glob Food Sec 2012:1:120-5.

32. Pérez-Escamilla R, Gubert MB, Rogers B, et al. Food security measurement and governance: Assessment of the usefulness of diverse food insecurity indicators for policy makers. Glob Food Sec 2017;14:96-104.

33. Haynes SN, Richard DCS, Kubany ES. Content validity in psychological assessment: a functional approach to concepts and methods. Psychol Assess 1995;7:238-47.

34. Boateng GO, Neilands TB, Frongillo EA, et al. Best practices for developing and validating scales for public health and nutrition research: A Primer. Frontiers in Public Health, 2018:1-18.

35. Krumdieck NR, Collins SM, Wekesa P, et al. Household water insecurity is associated with a range of negative consequences among pregnant Kenyan women of mixed HIV status. J Water Health 2016;14:1028-31.

36. McCoach DB, Gable RK, Defining MJP. Measuring, and Scaling Affective Constructs. Instrument development in the affective domain. New York: Springer New York, 2013:33-90.

37. Webb P, Iskandarani M. Water insecurity and the poor: issues and research needs. $1998 \mathrm{http}: / /$ www.iatp.org/files/Water Insecurity_and_the_Poor_Issues_and_Resea.htm (cited 20 Apr 2016).
38. Ericsson KA, Simon HA. Verbal reports as data. Psychol Rev 1980;87:215-51.

39. Kim ES, Cao C, Wang Y, et al. Measurement invariance testing with many groups: a comparison of five approaches. Structural Equation Modeling: A Multidisciplinary Journal 2017;24:524-44.

40. Guadagnoli E, Velicer WF. Relation of sample size to the stability of component patterns. Psychol Bull 1988;103:265-75.

41. United Nations Statistics Division. Designing household survey samples: practical guidelines. Geneva, Switzerland: United Nations Statistics Division, 2005.

42. Collins SM, Young SL. The Household Water InSecurity Experiences (HWISE) Scale Study Manual [Internet]. 2018 https://doi.org/10. 21985/N2M10F

43. Cohen S, Kamarck T, Mermelstein R. A global measure of perceived stress. J Health Soc Behav 1983;24:385-96.

44. Brewis A, Rosinger A, Wutich A, et al. Household water sharing favors charitable transfers to neighbors: evidence from eight waterinsecure sub-saharan african sites. Under review.

45. Brunette W, Sundt M, Dell N, et al. Open data kit 2.0: expanding and refining information services for developing regions. Proceedings of the 14th Workshop on mobile computing systems and applications: ACM, 2013:10

46. Cappelleri JC, Jason Lundy J, Hays RD. Overview of classical test theory and item response theory for the quantitative assessment of items in developing patient-reported outcomes measures. Clin Ther 2014;36:648-62.

47. Harvey RJ, Hammer AL. Item Response Theory. Couns Psychol 1999;27:353-83.

48. Raykov T, Marcoulides GA. Introduction to psychometric theory. New York, NY: Routledge 2011:335.

49. Wright BD, Stone M. Best Test design: rasch measurement. Chicago, IL: Mesa Press, 1979.

50. Smith EV. Evidence for the reliability of measures and validity of measure interpretation: a Rasch measurement perspective. J Appl Meas 2001;2:281-311

51. Linacre JM, Wright BD. A user's guide to BIGSTEPS: Rasch-model computer program. Chicago: Mesa Press, 1993.

52. Byrne BM, Watkins $D$. The Issue of measurement invariance revisited. J Cross Cult Psychol 2003;34:155-75.

53. Harkness JA. Vijver FJR van de, Mohler PP, editors. Cross-cultural survey methods. Hoboken, N.J: J. Wiley, 2003.

54. Leroy JL, Ruel M, Frongillo EA, et al. Measuring the food access dimension of food security: a critical review and mapping of indicators. Food Nutr Bull 2015;36:167-95.

55. Sideridis GD, Tsaousis I, Al-harbi KA. Multi-population invariance with dichotomous measures: combining multi-group and mimic methodologies in evaluating the general aptitude test in the arabic language. J Psychoeduc Assess 2015;33:568-84.

56. Asparouhov T, Muthén B. Multiple-group factor analysis alignment. Structural Equation Modeling: A Multidisciplinary Journal 2014;21:495-508.

57. Kim ES, Yoon M. Testing measurement invariance: a comparison of multiple-group categorical CFA and IRT. Structural Equation Modeling: A Multidisciplinary Journal 2011;18:212-28.

58. Lomazzi V. Using Alignment Optimization to test the measurement invariance of gender role attitudes in 59 Countries. Methods, data, analyses 2018;12 http://mda.gesis.org/index.php/mda/article/view/ 2017.09

59. Cronbach LJ. Coefficient alpha and the internal structure of tests. Psychometrika 1951;16:297-334.

60. Fowler FJ. Improving survey questions: design and evaluation. Thousand Oaks: Sage Publications, 1995.

61. Hadley C, Freeman MC. Assessing reliability, change after intervention, and performance of a water insecurity scale in rural Ethiopia. Food Secur 2016;8:855-64.

62. Angoff W. Use of difficulty and discrimination indices for detecting item bias. Handbook of methods for detecting test bias. Baltimore: Johns Hopkins University Press, 1982:96-116.

63. Gregorich SE. Do self-report instruments allow meaningful comparisons across diverse population groups? Testing measurement invariance using the confirmatory factor analysis framework. Med Care 2006;44(11 Suppl 3):S78. 
Correction: Development and validation protocol for an

instrument to measure household water insecurity across

cultures and ecologies: the Household Water InSecurity

Experiences (HWISE) Scale

Young SL, Collins SM, Boateng GO, et al. Development and validation protocol for an instrument to measure household water insecurity across cultures and ecologies: the Household Water InSecurity Experiences (HWISE) Scale. BMJ Open 2019;9:e023558. doi: 10.1136/bmjopen-2018-023558

This article was previously published with an error.

Four names were missed in the collaborators list. The names are Jonathan Maupin, Monet Niesluchowski, Asiki Gershim, and Divya Krishnakumar.

Open access This is an open access article distributed in accordance with the Creative Commons Attribution 4.0 Unported (CC BY 4.0) license, which permits others to copy, redistribute, remix, transform and build upon this work for any purpose, provided the original work is properly cited, a link to the licence is given, and indication of whether changes were made. See: https://creativecommons.org/licenses/by/4.0/.

C Author(s) (or their employer(s)) 2019. Re-use permitted under CC BY. Published by BMJ.

BMJ Open 2019;9:e023558corr1. doi:10.1136/bmjopen-2018-023558corr1

A) Check for updates 